\title{
Interleaved Transcranial Magnetic Stimulation/Functional MRI Confirms that Lamotrigine Inhibits Cortical Excitability in Healthy Young Men
}

\author{
Xingbao Li*,', Charlotte C Tenebäck', Ziad Nahas', F Andrew Kozel', Charles Large ${ }^{2}$, Jeffrey Cohn ${ }^{3}$, \\ Daryl E Bohning' and Mark S George' \\ 'Brain Stimulation Laboratory, Center for Advanced Imaging Research (CAIR), Medical University of South Carolina (MUSC), SC, USA; \\ ${ }^{2}$ Department of Neuropharmacology GlaxoSmithKline S.p.A., Verona, Italy; ${ }^{3}$ GlaxoSmithKline Inc., Research Triangle Park (RTP), NC, USA
}

\begin{abstract}
Little is known about how lamotrigine (LTG) works within brain circuits to achieve its clinical effects. We wished to determine whether the new technique of interleaved transcranial magnetic stimulation (TMS)/functional magnetic resonance imaging (fMRI) could be used to assess the effects of LTG on activated motor or prefrontal/limbic circuits. We carried out a randomized, double-blind, crossover trial involving two visits I week apart with TMS measures of cortical excitability and blood oxygen level-dependent TMS/fMRI. Subjects received either a single oral dose of $325 \mathrm{mg}$ of LTG or placebo on each visit. In all, I 0 subjects provided a complete data set that included interleaved TMS/FMRI measures and resting motor threshold (rMT) determinations under both placebo and LTG conditions. A further two subjects provided only rMT data under the two drug conditions. LTG caused a $14.9 \pm 9.6 \%$ (mean \pm SD) increase in rMT $3 \mathrm{~h}$ after the drug, compared with a $0.6 \pm 10.9 \%$ increase $3 \mathrm{~h}$ after placebo $(t=3.4 \mathrm{I}, \mathrm{df}=1 \mathrm{I}, p<0.0 \mathrm{I})$. $\mathrm{fMRI}$ scans showed that LTG diffusely inhibited cortical activation induced by TMS applied over the motor cortex. In contrast, when TMS was applied over the prefrontal cortex, LTG increased the TMS-induced activation of limbic regions, notably the orbitofrontal cortex and hippocampus. These results suggest that LTG, at clinically relevant serum concentrations, has a general inhibitory effect on cortical neuronal excitability, but may have a more complex effect on limbic circuits. Furthermore, the interleaved TMS/fMRI technique may be a useful tool for investigating regional brain effects of psychoactive compounds.

Neuropsychopharmacology (2004) 29, 1395-1407, advance online publication, 2 I April 2004; doi: I 0. I038/sj.npp. I 300452
\end{abstract}

Keywords: transcranial magnetic stimulation; neuroimaging; lamotrigine; motor cortex; prefrontal cortex; healthy young men

\section{INTRODUCTION}

Lamotrigine (LTG) is a use-dependent sodium channel inhibitor with broad-spectrum anticonvulsant efficacy against a range of epilepsy syndromes (Messenheimer 1995). Recently, several double-blind, placebo-controlled trials have demonstrated the acute and prophylactic antidepressant activity of LTG in bipolar disorder (Calabrese, 1999; Calabrese and Gajwani, 2000; Frye et al, 2000). Anticonvulsant mood stabilizers may work through the same mechanisms needed for seizure control, but in

\footnotetext{
* Correspondence: Dr X Li, Brain Stimulation Laboratory, Center for Advanced Imaging Research (CAIR), Medical University of South Carolina (MUSC) IOP, 502 N, 67 President St, Charleston, SC 29425 , USA, Tel: + I 843876 5I42, Fax: + I 843792 5702,

E-mail: lixi@musc.edu

Received 08 August 2003; revised 27 January 2004; accepted 26 February 2004

Online publication: 03 January 2004 at http://www.acnp.org/citations/ Npp03010403362/default.pdf
}

different brain regions. Thus, some have suggested that LTG stabilizes mood by reducing cortical excitability in areas relevant to the pathogenesis of mood disorders (Ketter and Calabrese, 2002).

Transcranial magnetic stimulation (TMS) is a noninvasive means to stimulate the cerebral cortex as well as to assess the motor cortex excitability (George et al, 1999; Ziemann et al, 1996). TMS has been used to examine the pharmacologic effects of anticonvulsant drugs on the excitability of motor corticospinal pathways in both patients with epilepsy and normal subjects (Boroojerdi et al, 2001; Manganotti et al, 1999; Ziemann et al, 1996, 1998b, 2002). In volunteers or patients with complex partial seizures, LTG significantly increased the resting motor threshold (rMT) (Manganotti et al, 1999; Ziemann et al, 1996, 1998b). Thus, TMS combined with motor-evoked potential (MEP) measurements can provide useful information about medication effects, but the information is limited to assessing how the drug affects motor circuits. TMS over nonmotor brain areas does not produce an easily observable 
behavioral response, so TMS alone cannot provide information about medication effects in these other important brain regions.

Combining TMS with noninvasive imaging techniques allows one to observe TMS effects throughout the brain. Initial studies used fluorodeoxyglucose (George et al, 1995; Kimbrell et al, 1999) or oxygen (O15) (Paus et al, 1997; Paus and Wolforth, 1998) positron emission tomography (PET). Our group at the Medical University of South Carolina (MUSC) pioneered and developed a technique for interleaving TMS with blood oxygen level-dependent (BOLD) functional magnetic resonance imaging (fMRI) (Bohning et al, 1999; Shastri et al, 1999). Another group has now succeeded in performing this technique (Baudewig et al, 2001; Bestmann et al, 2003).

To our knowledge, no one has used interleaved TMS/ fMRI yet to examine medication effects. In the present study, we used interleaved TMS/fMRI to image brain activity during TMS over the motor cortex and prefrontal cortex in healthy subjects after receiving a single oral dose of LTG or placebo. We sought to compare rMT and the BOLD TMS-induced pattern of brain activation after LTG or placebo. We hypothesized that, compared to placebo, a single oral dose of LTG would inhibit brain excitability. This LTG effect would be seen as increased rMT and reduced TMS-induced BOLD activation of the motor cortex. We further speculated that LTG would inhibit TMS-induced brain activation of cortical and limbic regions during TMS over the prefrontal cortex. This proof-of-concept study sought to test specifically whether interleaved TMS/fMRI might prove a useful tool in understanding LTG's moodstabilizing mechanism of action. We also sought to understand whether the interleaved technique might be of general use in the study of psychoactive substances.

\section{SUBJECTS AND METHODS}

\section{Subjects}

All subjects included in this study were given a detailed explanation of the procedure and signed a consent form approved by the MUSC Institutional Review Board (IRB) and the Food and Drug Administration (FDA). In all, 14 healthy young men (aged 18-30 years) were recruited by local advertisement and then had a screening history and physical examination, structured diagnostic interview (First et al, 1995), baseline laboratory work (basic metabolic panel, liver panel, and hematology), and urine drug screen for drugs of abuse. All subjects were right-handed determined by the Annett Handedness Questionnaire (Annett 1970) and were nonsmokers. We restricted this initial study to men, as there are known variations in the TMS rMT as a function of menstrual cycle stage (Smith et al, 2002).

\section{Procedures}

Study design: We performed a randomized, double-blind, crossover trial involving two visits at least 1 week apart. The subjects received either a single oral dose of $325 \mathrm{mg}$ of LTG or placebo on the first visit, and then on the second visit they were given whatever they did not initially receive. A single oral dose of $325 \mathrm{mg}$ of LTG has been shown to produce, at $3 \mathrm{~h}$, serum concentrations equal to steady-state levels at clinically relevant chronic doses (Tergau et al, 2003).

Visit protocol: (1) We measured predose rMT with a MAGSTIM stimulator placed over the left motor cortex and obtained blood for baseline plasma levels of LTG. (2) Subjects took a single oral dose of $325 \mathrm{mg}$ LTG or placebo, and then waited $3 \mathrm{~h}$. (3) We measured postdose rMT with the same MAGSTIM stimulator and drew blood to determine postdose serum plasma levels of LTG. (4) Subjects then walked to the MRI suite where we measured rMT with a specially modified Dantec MagPro stimulator. (5) We performed interleaved TMS/fMRI scanning with the TMS coil positioned over the left motor cortex. (6) Finally, we performed interleaved TMS/fMRI scanning with the TMS coil positioned over the left prefrontal cortex.

After 1 week, they received LTG or placebo (whichever they had not received during the first visit) followed by identical rMT and interleaved TMS/fMRI studies.

\section{rMT Measures}

TMS: In the Brain Stimulation Laboratory, focal TMS was delivered by a figure-of-eight magnetic coil (each wing $70 \mathrm{~mm}$ in diameter) connected to a MAGSTIM Super Rapid stimulator (Magstim Co., Whitland, Dyfed, UK), which generates biphasic electrical pulses of approximately $250 \mu \mathrm{s}$ duration. The optimal position of the magnetic coil for eliciting an MEP in the right abductor pollicis brevis (APB) was determined by holding the coil tangential to the scalp and moving it in small steps over the presumed area of the left primary motor cortex at a slightly suprathreshold stimulus intensity. The coil was always held horizontally with the handle pointing backward and laterally at $45^{\circ}$ from the midline. This position was marked with a pen on a reusable latex swimming cap in order to assure constant placement of the coil throughout the visits. Stimulus intensity and threshold values were expressed as the percent of the maximal stimulator output.

$r M T$ : Surface electromyograms (EMG) were recorded from the APB using 9-mm Ag-AgCl electrodes in a bellytendon montage. The placement of electrodes on the thumb and hand was marked with a pen for exact replacement for subsequent tests during the visit. The raw EMG signal was amplified by a factor of 100 and band-pass filtered, $70 \mathrm{~Hz}$ to $2.0 \mathrm{kHz}$, with a High Performance Band Pass Filter, Model V-75-48 (LAB Linc. Co.). The EMG was recorded on a G3 Macintosh with MacCRO (version 2.1) software.

rMT was determined in the resting APB in four steps: in steps one and three, thresholds were approached from a slightly suprathreshold intensity by reducing the stimulus intensity in $1 \%$ steps with a $5 \mathrm{~s}$ interval between pulses, whereas in steps two and four, thresholds were approached from a slightly subthreshold intensity by increasing the stimulus intensity in $1 \%$ steps. rMT was defined as the lowest intensity that produced an MEP of greater than $50 \mu \mathrm{V}$ in three out of six trials in the resting target muscle (Mills and Kimiskidis, 1996). A mean rMT at baseline or after medication was calculated by averaging the four values. Determination of the rMT using this technique usually took $30 \mathrm{~min}$. 


\section{Interleaved TMS/fMRI Methods}

Combined TMS and MRI: TMS was applied within the MRI scanner through a Dantec MagPro, which generates biphasic electrical pulses of approximately $200 \mu$ s duration (Dantec Medical A/S, Skovlunde, Denmark). The pulses were delivered through a special nonferromagnetic TMS coil of figure-of-eight (each wing $100 \mathrm{~mm}$ in diameter) design with an $8-\mathrm{m}$ cable and a room setup identical to prior TMS/ fMRI studies from our group. TMS pulses and the fMRI sequence were interleaved as described before (Shastri et al, 1999).

TMS coil placement in the MRI scanner. Motor cortex: For technical reasons, we were unable to use the Magstim within the MRI scanner room and instead used a Dantec TMS with a different coil for interleaved TMS/fMRI. Since rMT is likely to differ slightly from machine to machine (due to different capacitors, coil design, length of cable, MRI filter, etc), subjects were retested using the Dantec TMS before being placed into the MRI scanner. This rMT measure was used to determine stimulation amplitude during the subsequent interleaved TMS/fMRI session. The previous rMT, measured in the Brain Stimulation Laboratory using the Magstim, was used for analysis of the effects of drug on rMT. After this new MRI rMT was determined, the TMS coil was rigidly mounted in the MR head coil with a specially designed TMS coil holder, adjustable in six dimensions (Bohning et al, 2003). Subjects wore swim caps and special earplugs. With the head coil on the gantry outside the scanner bore, subjects inserted their head into the head coil and adjusted their position, while the TMS coil was intermittently pulsed with $100 \%$ rMT. Subjects adjusted their head until pulsing the coil caused visible movement of the contralateral (right) hand APB (three out of six). As soon as a subject's correct scalp location was determined, the holder's six dimensions and earplugs were locked. These head holder settings and rMT were recorded and used also for the second visit. Immediately after the motor cortex MRI study, subjects were removed from the scanner and the TMS device was moved to a position over the left prefrontal cortex. The left prefrontal cortex stimulation site was defined as a location $5-\mathrm{cm}$ rostral and in a parasagittal plane from the site of maximal APB stimulation. Subjects then re-entered the scanner for the prefrontal TMS scan.

During the second MRI visit a week later, the head holder was set with the previous week's coordinates for that subject and the previous rMT was used for the second visit.

fMRI was performed in a Picker EDGE 1.5 MR scanner (Picker International, Inc., Cleveland, OH). Each fMRI acquisition was a gradient echo planar imaging scan with 15 slices and 294 time points, TE/TR $=40 / 3000 \mathrm{~ms}$ with $90^{\circ}$ flip, $128 \times 128$ matrix on $27 \mathrm{FOV}$, slice thickness $7 \mathrm{~mm}$ and gap $1 \mathrm{~mm}$. The total acquisition time was $14.7 \mathrm{~min}$. Two acquisitions were made, one with TMS over the motor cortex and one with TMS over the prefrontal cortex. Each acquisition included a total of 294 time points divided into seven repeating cycles; each cycle consisted of six segments as follows: rest (no TMS), 100\% TMS, rest, rest, $120 \%$ TMS, rest. Each segment consisted of seven time points.

\section{Image Data Analysis}

Individual fMRI data analyses: MR scans were transferred into ANALYZE format and then further processed on Sun workstations (Sun Microsystems, Palo Alto, CA). Scans were checked using MEDx3.3 (Sensor Systems Inc., Sterling, VA) for movement across runs, and then were coregistered to a mean image using automatic image registration. For all subjects, movement across the 14.7-min acquisition was less than $2 \mathrm{~mm}$ in all three axes. After correction for motion, we used a delayed boxcar model, employing a high-pass filter to remove signal drift, cardiac and respiratory effects, and other low-frequency artifacts. Then, we transformed each subject's data into Talairach space (input voxel dimensions, $2.1 \times 2.1 \times 8 \mathrm{~mm}^{3}$, to output voxel dimensions, $4 \times 4 \times$ $\left.4 \mathrm{~mm}^{3}\right)$, smoothed $\left(4 \times 2 \mathrm{~mm}^{2}\right)$ the data, and generated $\mathrm{z}$ maps with the Statistical Parametric Mapping (SPM) 96 module in MEDx3.3. We assumed an uncorrected $F$ threshold UF $p>0.99$ to preserve as many voxels as possible for the cluster analysis. Only clusters showing a statistical weight of $p<0.05$ were considered to be significantly activated.

Group fMRI data analyses: Unthresholded $\mathrm{z}$ maps from all the subjects were combined based on comparison of condition (100\% rMT stimulation $v s$ rest and $120 \% \mathrm{rMT}$ stimulation $v s$ rest), intensity (100\% rMT-TMS $v s \quad 120 \%$ rMT-TMS), and visit (LTG vs placebo). The combined group $z$ maps were thresholded using $z \geqslant 3.09$. $(p<0.001)$ and cluster statistical weight (spatial extent threshold) of $p<0.05$. We used either paired or unpaired $t$-tests in MEDx3.3 for all comparisons of interest and both areas of stimulation.

Magnitude of BOLD time-course response: To compare the magnitudes of BOLD signal changes, two types of data were recorded. The different maps of LTG and placebo were used to make a mask of the left motor cortex (82 voxels) and a mask of the left hippocampus (19 voxels) (Figure 2 bottom panels). The masks used to define location were taken as an index of relative peak intensity above noise. According to the masks' Talairach coordinates, the mean signal intensity of the highest six contiguous voxels (two in each slice) in each subject was extracted from the motor cortex or hippocampus with SPM plotting in MEDx. The cycle-mean time series determined for each subject were transferred to a spreadsheet program and, by averaging point-by-point within and across subjects, subject-mean and grand-mean time series were determined ( $\%$ signal change $=100$ (mean signal at each point-averaged signal in all preceding rests)/ averaged signal in all preceding rests).

\section{Statistical Analysis on Other Variables}

The percent change of rMT was calculated as follows: \% change $=100($ (postdrug $\mathrm{rMT}-$ predrug $\mathrm{rMT}) /$ predrug rMT). Paired Student's $t$-tests (two tailed) were performed for the percent change of rMT between LTG and placebo. Wilcoxon's nonparametric tests were performed for the number of active voxels in the region of interests (ROI) between LTG and placebo. We performed Pearson's correlations between the percent change of rMT and the change of active voxel number. Two-way analysis of variance (ANOVA) was performed for \% BOLD signal 
change under the different intensity stimulation conditions and the different medication conditions. All statistical analyses were performed using SPSS 10.0 (Statistical Product and Service Solutions Inc., Chicago IL).

\section{RESULTS}

In all, 14 subjects were enrolled and were studied. Technical problems with the fMRI scanner or TMS machines meant that not all subjects provided complete data sets. One subject had a baseline rMT greater than the Magstim machine output. After we determined this, the subject was not studied further. Of the 13 subjects studied on 2 days, 12 subjects (age $25.31 \pm 2.70$ years) had usable paired TMS/ rMT data, and of these, two subjects completed the protocol, but their MRI data on at least one of the visits were not usable because of MRI scanner problems. Thus, 10 subjects had complete placebo and LTG interleaved TMS/ fMRI data as well as complete rMT data. LTM serum concentration $(n=10)$ was $3.473 \pm 0.414 \mu \mathrm{g} / \mathrm{ml} \quad$ (range 4.151-2.809).

\section{Safety and Tolerability}

None of the subjects reported experiencing adverse effects of the drug treatment or the stimulation.

\section{Effects of LTG on rMT}

Consistent with our prestudy hypothesis, LTG elevated mean rMT significantly by $14.9 \pm 9.6 \%$ from the same day baseline compared with a placebo increase over baseline
$0.6 \pm 10.9 \%$ (paired Student's $t$-test, $t=3.41, \mathrm{df}=11$, $p<0.01$ ) (see Table 1 and Figure 1).

Correlation analyses and $t$-tests were performed on the rMT data between visits to assess the repeatability of the rMT and the natural variation. The predrug rMT on visit 1 correlated with the predrug rMT on visit $2(r=0.84, n=12$, $p<0.01)$, and paired $t$-test results showed no significant difference between them $(t=0.87, \mathrm{df}=11, p>0.05)$, indicating good reliability of the rMT within subjects across visits 1 week apart. On both visits, the predrug rMT

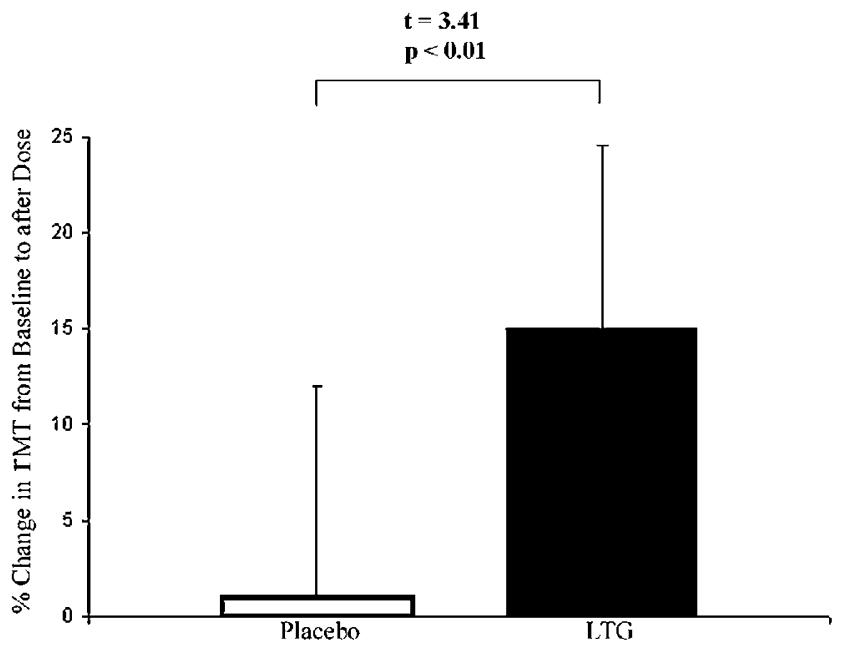

Figure I Comparison of the changes of rMT between LTG and placebo. TMS rMT data for all 12 subjects showed a significant increase from baseline on the day that subjects received LTG compared with placebo (Student's $t$-test, $t=3.4 \mathrm{I}, \mathrm{df}=\mathrm{II}, p<0.0 \mathrm{I}$ ).

Table I rMT and \% Change from Baseline in 12 Subjects on the Two Different Visits (Placebo, LTG)

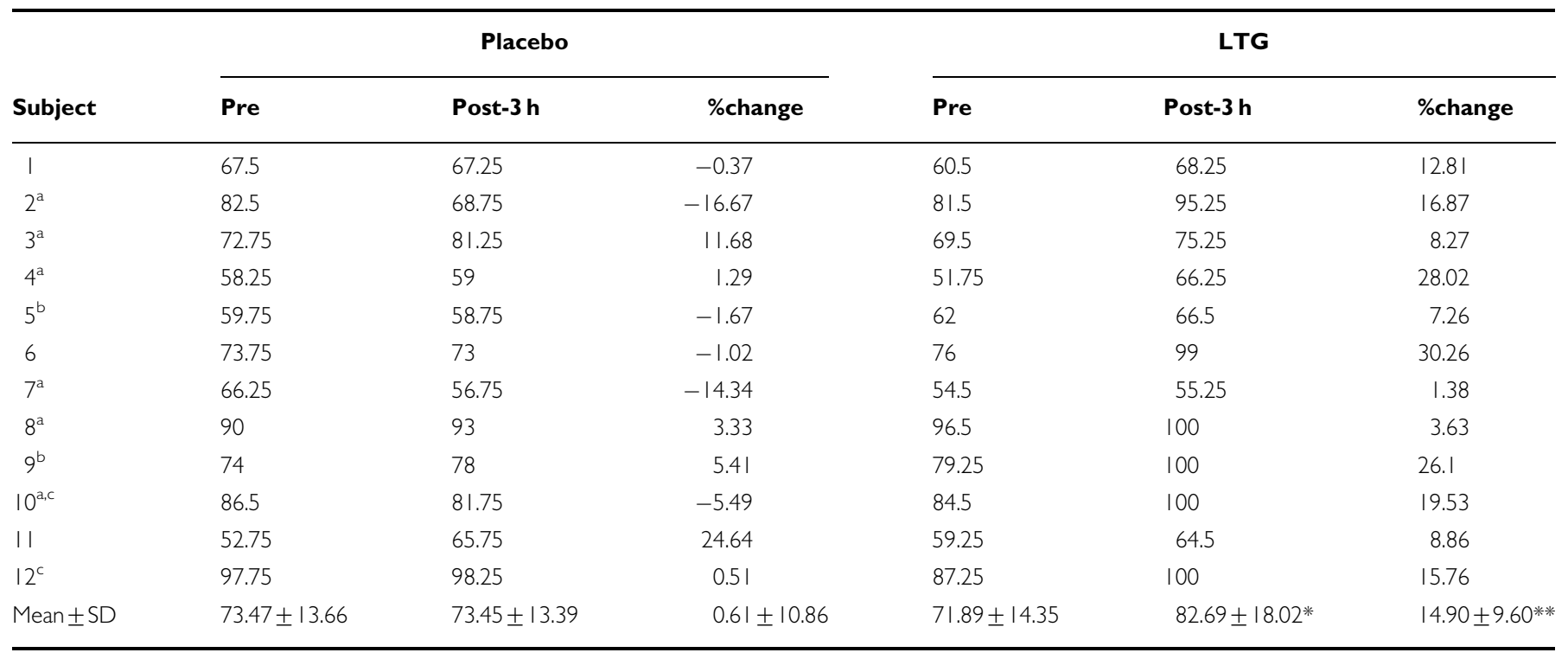

Units are percent of machine maximum output (Magstim).

* $t=5.20, p<0.01$ compared with pre-LTG.

*** $t=3.4 \mathrm{I}, p<0.0$ I compared with placebo.

aisit I-LTG.

bNo fMRI data.

${ }^{\mathrm{C}}$ On LTG, rMT above $100 \%$ of the maximum output. 
correlated well with the postdrug rMT. On the LTG day, the correlation was shifted, with higher rMT following LTG (placebo visit: $r=0.89, n=12, p<0.01$; LTG visit: $r=0.86$, $n=12, p<0.01)$. However, we failed to find a correlation between the serum levels of LTG and postdrug rMT ( $r=0.34, n=12, p=0.33)$. rMT data assessed by the Dantec machine within the scanner $(80.17 \pm 12.98)$ were consistent with the data obtained by the Magstim machine in the Brain Stimulation Laboratory $(81.50 \pm 17.59)$. rMT at the first visit before drug dosing assessed by the Magstim significantly correlated with that obtained by the Dantec on the first visit, regardless of dose $(r=0.87, n=12, p<0.01)$.

\section{BOLD Response to TMS}

Motor cortex stimulation: For the motor cortex stimulation, six unthresholded $\mathrm{z}$ maps were obtained from each subject; these were $100 \%$ rMT vs rest, $120 \%$ rMT vs rest, and $100 \%$ rMT vs $120 \%$ rMT in the presence of placebo or LTG. These $\mathrm{z}$ maps were used as intermediate data for the group analysis.

The motor cortex TMS after either placebo or LTG (within-day analysis) at both $100 \%$ rMT and 120\% rMT resulted in diffuse activation in the brain, including the motor cortex underneath the TMS coil (see Table 2).

Prefrontal cortex stimulation. Eight of the 10 subjects provided usable data from the prefrontal interleaved TMS/ fMRI visits. Two subjects whose results were included in the motor cortex analysis could not be used in the prefrontal analysis because there was evidence of head movement of more than $2 \mathrm{~mm}$ between time points during the prefrontal TMS session.

With the prefrontal cortex stimulation, again six unthresholded $\mathrm{z}$ maps were obtained from each subject; as before, these were $100 \%$ rMT $v s$ rest, $120 \%$ rMT $v s$ rest, and $100 \%$ rMT vs $120 \%$ rMT in the presence of placebo or LTG. These $\mathrm{z}$ maps were used as intermediate data for group analysis.

The prefrontal cortex stimulation compared to rest, after either placebo or LTG, at both $100 \%$ rMT and $120 \%$ rMT stimulation, induced activation in diffuse brain regions. Of particular note, brain activity was not significantly increased from rest at the site of stimulation immediately underneath the coil with either $100 \%$ rMT or $120 \%$ rMT stimulation (see Table 3 and Figure 2).

\section{Intensity-Dependent BOLD Response Induced By TMS}

Motor cortex stimulation: On the placebo day, motor cortex TMS data showed that $120 \%$ rMT stimulation caused more activation than did $100 \%$ rMT stimulation in several regions including underneath the coil (see Table 2). While, on the LTG day, no significant difference was found between $100 \%$ rMT and $120 \%$ rMT motor cortex stimulation (Table 2).

Prefrontal cortex stimulation. In contrast to the motor cortex stimulation, and independent of the presence of placebo or LTG, there were no statistically significant differences in the pattern of activation between $100 \%$ rMT and $120 \%$ rMT over the prefrontal cortex stimulation (Table 3).

\section{Effects of LTG on BOLD Response Induced by TMS}

Motor cortex stimulation: At $120 \%$ rMT stimulation, a between-day analysis revealed that, compared to placebo, on the day subjects were taking LTG, they had significantly less TMS-induced activation in the motor cortex (underneath the coil), posterior cingulate, precuneus, and cerebellum (see Figure 2 bottom panel and Table 4). However, no similar findings were found at $100 \% \mathrm{rMT}$ stimulation.

Prefrontal cortex stimulation: A between-day analysis showed that, with respect to the rest condition, there was increased brain activity in the hippocampus and the orbital frontal gyrus during $100 \%$ rMT stimulation in the presence of LTG compared to placebo (Figure 2 bottom panel, Table 4). However, in this case no similar findings were found at $120 \%$ rMT stimulation.

\section{Post hoc Assessments}

As a further check on the effects of LTG on brain activity described above, we examined the number of voxels and the time series of activation of voxel clusters in the motor cortex (120\% rMT stimulation) and hippocampus (100\% rMT stimulation).

The number of voxels: The number of voxels that were significantly activated by $120 \%$ rMT TMS (compared to rest) over the motor cortex, during the placebo and LTG conditions, is shown in Figure 3 for each of the 10 subjects. LTG significantly decreased the number of voxels activated by $120 \%$ rMT TMS in the motor cortex. Furthermore, in order to test whether the brain imaging results corresponded with the electrophysiological measures, Pearson's correlations were performed on the relationship between the rMT before and after administration of LTG, and the number of active voxels underneath the coil between LTG and placebo days. A significant correlation was found between the increased rMT and inhibited activation in the motor cortex $(n=10, r=0.81, p<0.01)$.

For the prefrontal cortex data, the number of voxels that were significantly activated by $100 \%$ rMT TMS (compared to rest) over the prefrontal cortex, during the placebo and LTG conditions, is shown in Figure 3 for each of the eight subjects. There were significantly more TMS-induced active voxels in the left hippocampus after LTG than after placebo.

Percent BOLD signal change: For the motor cortex, the cycle-averaged time-activity curve was plotted and an estimate was obtained of the level of activity in the $120 \%$ rMT TMS subcycle relative to the preceding rest subcycle. Figure 4 summarizes the time-activity data pooled across 10 subjects for the motor cortex stimulation. LTG dampened the TMS-induced BOLD response by approximately $50 \%$. Two-way ANOVA results showed that the \% BOLD signal change in the presence of LTG was significantly decreased compared with placebo $\left(\mathrm{F}_{1,20}=11.89, p=0.007\right)$, and the $\%$ BOLD signal change of $120 \%$ rMT was significantly increased compared with $100 \% \operatorname{rMT}\left(\mathrm{F}_{1,6}=6.27, p=0.034\right)$.

We also examined the time series of activation of the cluster of voxels in the left hippocampus (100\% rMT stimulation). Figure 4 summarizes the time-activity data pooled across eight subjects. Two-way ANOVA results of the entire time series failed to show significant differences 
Table 2 Active Regions during TMS Stimulation Over the Motor Cortex (Within-Day Analyses, $n=10$ ) Threshold for Inclusion, $Z \geqslant 3.09$ extent $p<0.05$

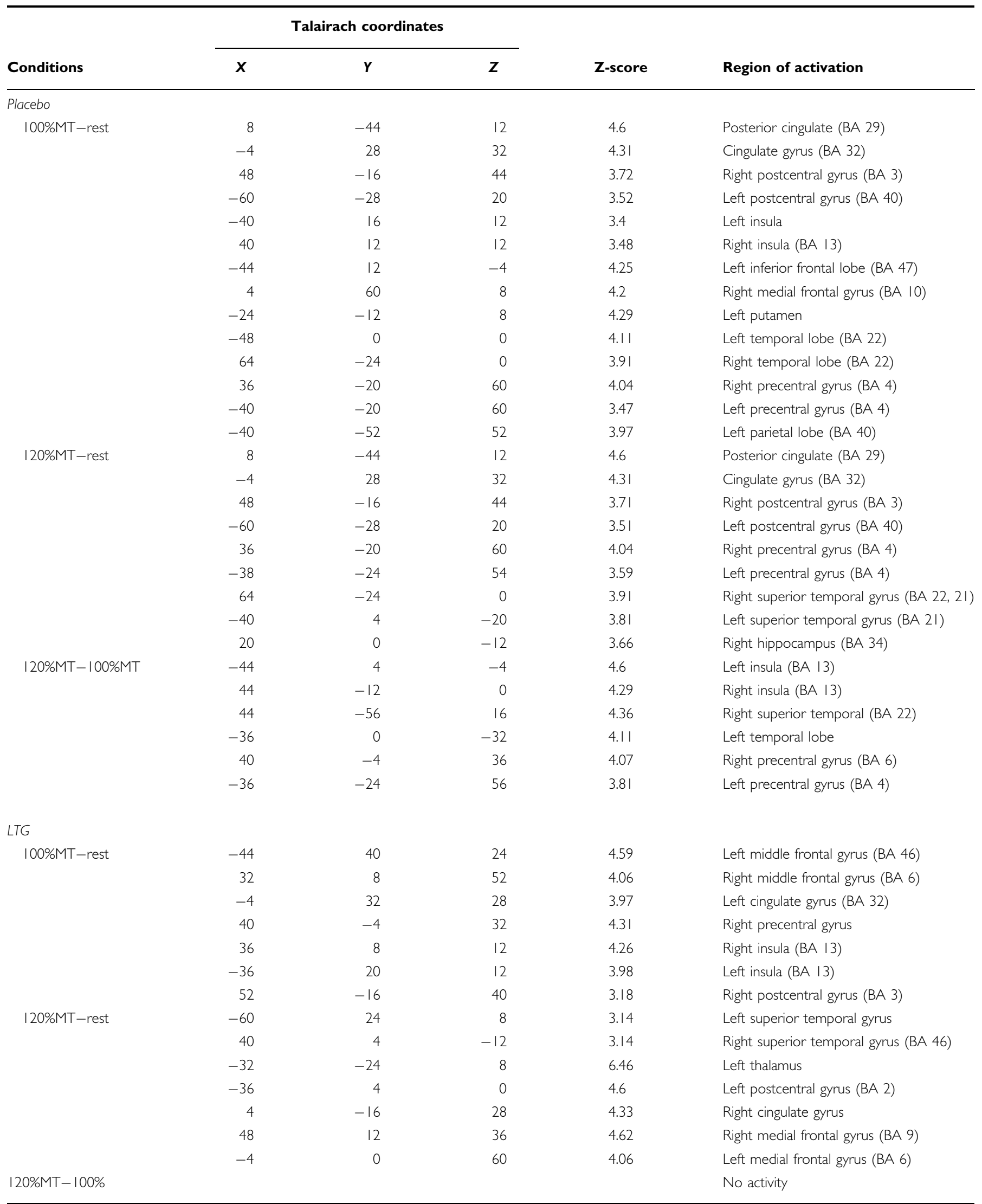


Table 3 Active Regions during TMS Stimulation Over the Prefrontal Cortex (Within-Day Analyses, $n=8$ ) Threshold for Inclusion, $Z \geqslant 3.09$ extent $p<0.05$

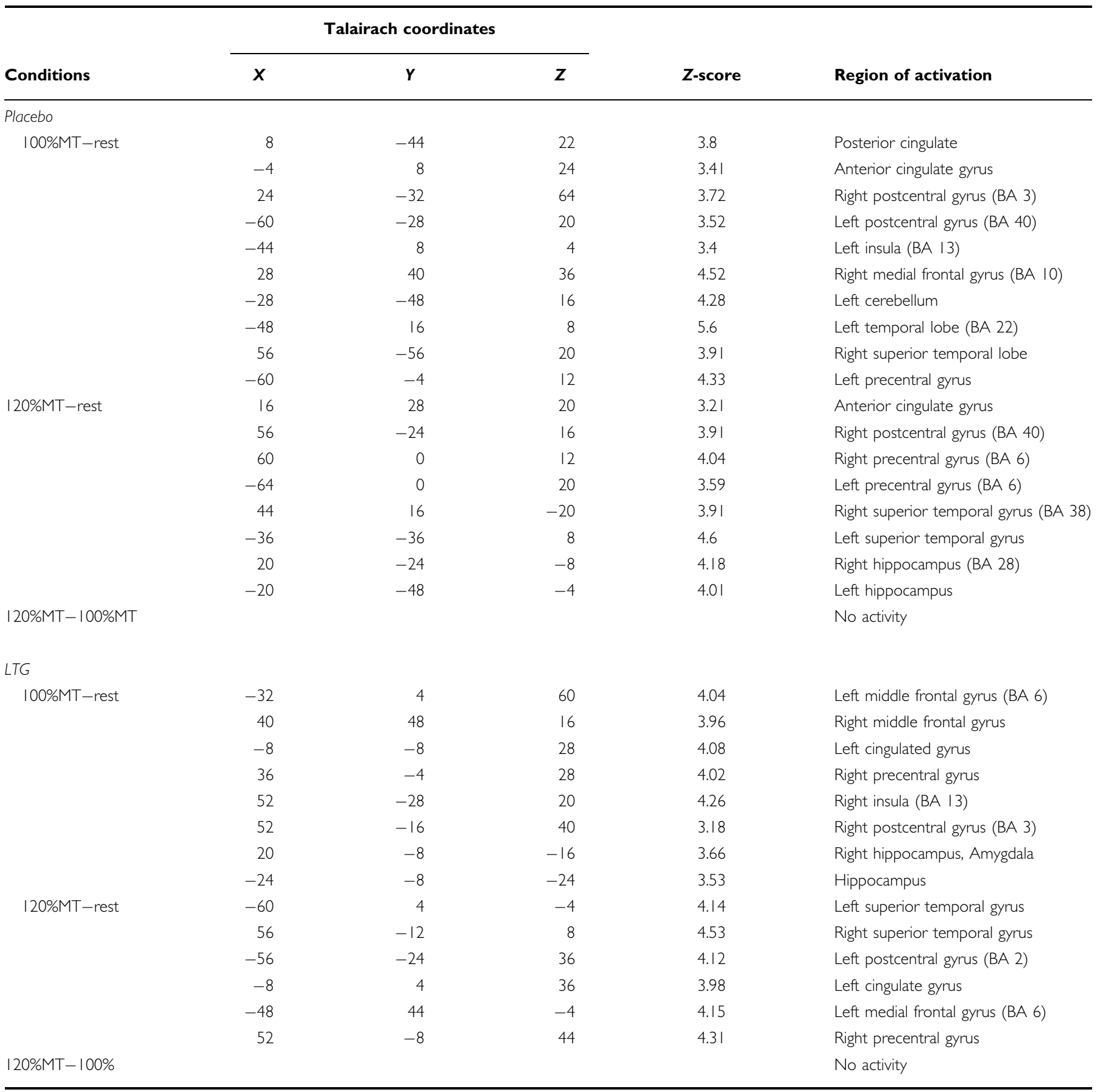

in \% BOLD signal change between LTG and placebo $\left(\mathrm{F}_{1,20}=1.12 p=0.326\right)$ or between $100 \%$ rMT stimulation and $120 \%$ rMT stimulation $\left(\mathrm{F}_{1,6}=0.32, p=0.591\right)$.

\section{DISCUSSION}

\section{Interleaved TMS/fMRI}

To our knowledge, this is the first report to use the interleaved TMS/fMRI technique to investigate the regional brain effects of a central nervous system (CNS)-active compound. We found, consistent with our hypothesis, that LTG inhibited the motor cortex activation that occurred when we applied TMS over this area to elicit a thumb movement. This LTG inhibition was evident both from the electrophysiological measurements of rMT and the regional BOLD response. Furthermore, with stimulation over the motor cortex, the brain imaging and electrophysiological measures were highly correlated. In contrast, when TMS was applied over the prefrontal cortex, we found that LTG 


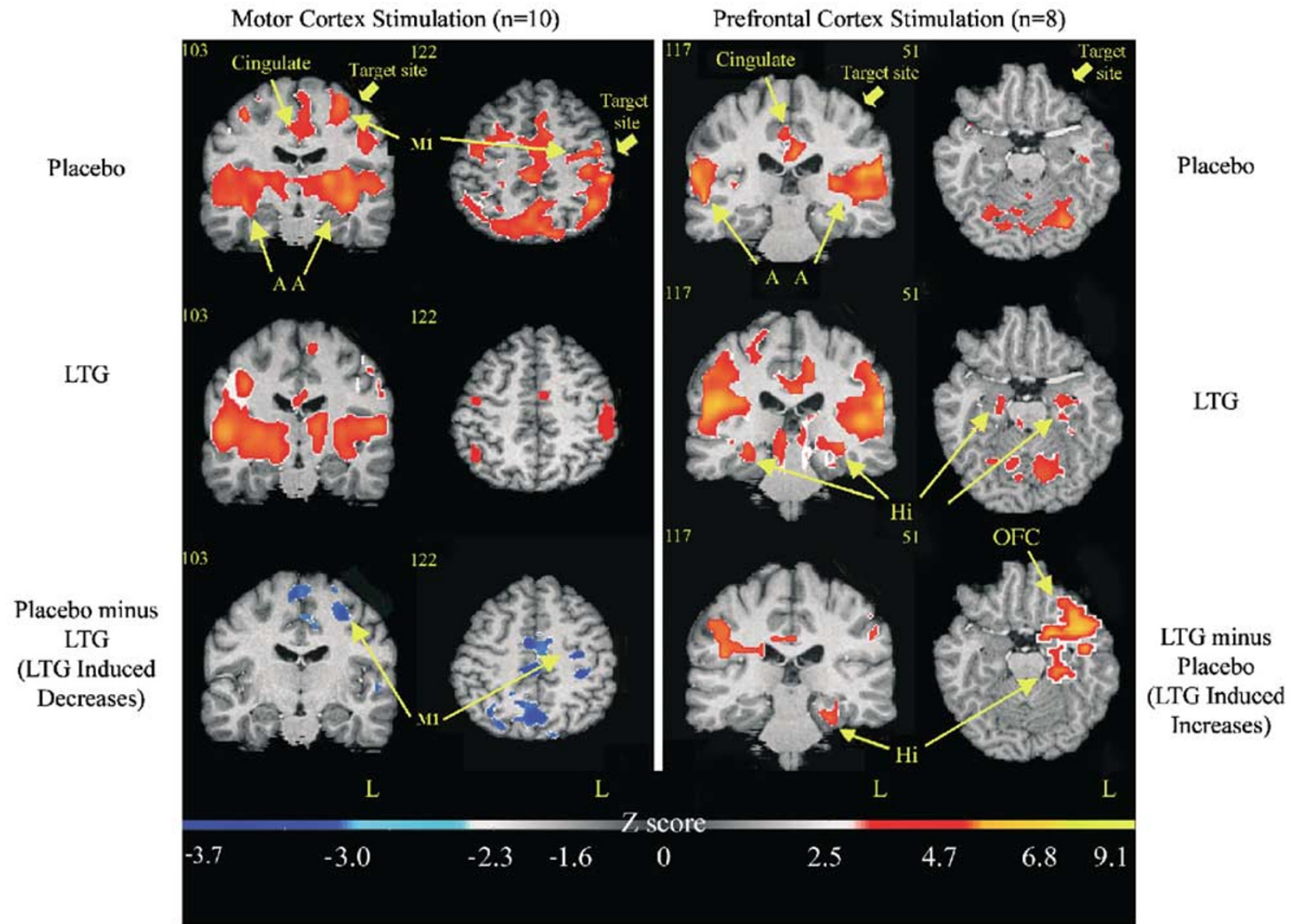

Figure 2 Effects of LTG on TMS-induced brain activation. These are the group data in 10 subjects for the motor cortex (left figure) and eight subjects for the prefrontal cortex stimulation (right figure). The group differences of TMS-rest are shown depicted on a representative brain in Talairach coordinates. On the left of the image are the results for TMS over the motor cortex stimulation at I20\% rMT for Placebo (top), LTG (middle), and the difference between LTG and placebo (bottom). Note that motor cortex TMS causes local and distant activation, and that LTG reduced this TMS-induced activity both locally underneath the coil and in connected regions. On the right of the image are the results for TMS over the prefrontal cortex stimulation at I00\% rMT for placebo (top), LTG (middle). The differences between LTG and placebo (bottom) are shown with a lower threshold of $p<0.05$, extent 0.05. Note that prefrontal cortex TMS causes limbic system activation in this study only when subjects were taking LTG. MI= motor cortex; Hi=hippocampus; $\mathrm{AA}=$ auditory area; $\mathrm{OFC}=$ orbitofrontal cortex; target site $=$ placement of TMS.

did not inhibit the BOLD response, but apparently increased the activation of the limbic system.

The results demonstrate that it is possible to combine TMS and fMRI to evaluate both decreasing and increasing regional brain effects of CNS compounds. Thus interleaved TMS/fMRI may be a useful new neuroscience tool and may have several important uses in the study of psychoactive drugs. This study also highlights the considerable additional work that will be required before this technique could be routinely applied in pharmacological testing and screening.

\section{BOLD Response to TMS Over the Motor Cortex}

Analysis of the group fMRI data from TMS over the motor cortex on the placebo day revealed robust TMS-induced activation of the ipsilateral motor cortex consistent with prior TMS/fMRI studies over the motor cortex (Baudewig et al, 2001; Bohning et al, 2000) as well as bilateral activation of the auditory cortex (Boroojerdi et al, 1999).
Interestingly, the present data also showed that TMS caused activation of the contralateral (right) motor cortex as well. Although the control of movement is one of the clearest hemispherically lateralized functions in the brain (Dassonville et al, 1998), human functional neuroimaging studies of hand motor control commonly report bilateral activation in the primary motor cortex (Hlustik et al, 2002; Solodkin et al, 2001). We also compared BOLD-fMRI responses at two different stimulation intensities and found that highintensity motor cortex stimulation (120\% rMT) was associated with significantly increased activation compared to lower intensity (100\% rMT) stimulation (Bohning et al, 2000 ), on the placebo day only. These results on the placebo medication day replicate our previous studies of motor cortex TMS/fMRI, all of which have shown intensitydependent TMS effects (Bohning et al, 2001, 1999). Finally, as a further check on our findings, we also analyzed the time series of activation in the motor cortex and found that a $1 \%$ BOLD activation relative to baseline could be observed with $120 \%$ rMT stimulation. 
Table 4 Talairach Coordinates of Regions Significantly Affected by LTG (Between-Day Analyses)

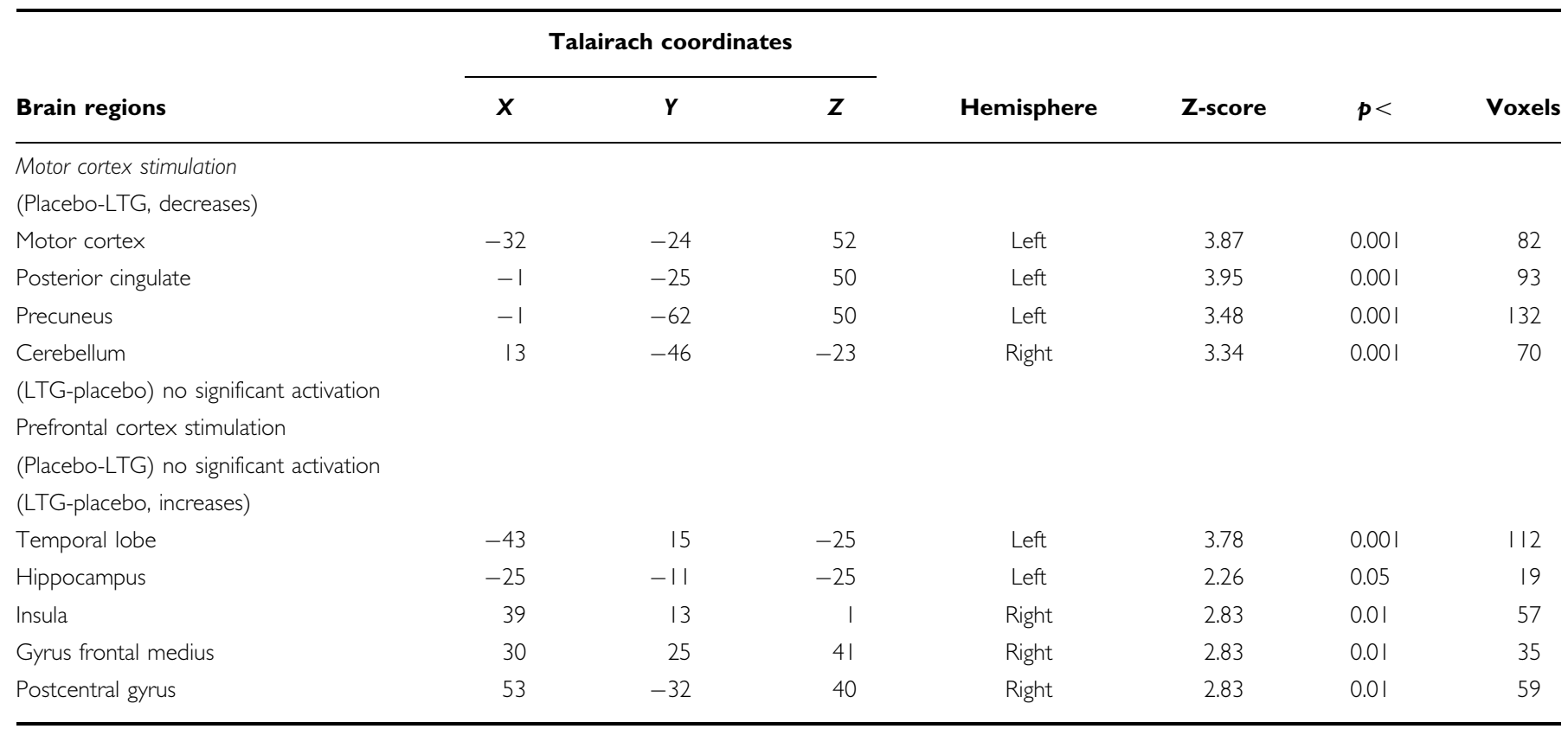
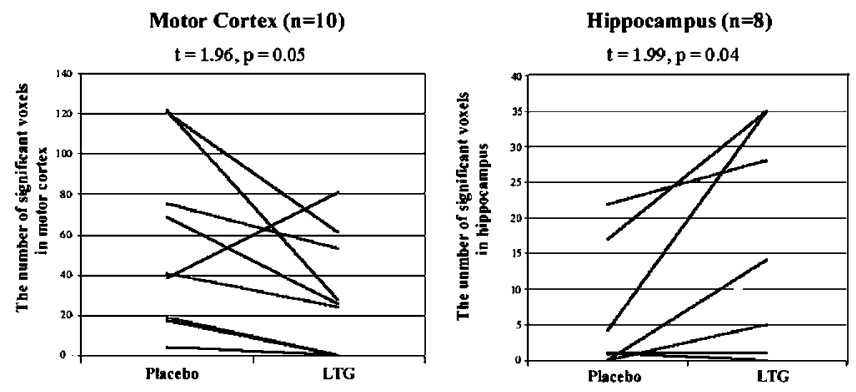

Figure 3 Changes of significant active voxel number by LTG in the motor cortex and hippocampus. The left panel depicts the number of significant voxels in each subject in an ROI directly underneath the TMS coil, during the motor cortex stimulation (I20\% rMT minus rest over the motor cortex) for the placebo day and the LTG day. Compared with placebo, LTG significantly decreased the number of active voxels in the motor cortex (Wilcoxon's nonparametric test: $z=1.96, \mathrm{df}=9, p=0.05$ ). The panel on the right depicts the number of significant voxels in individuals in an $\mathrm{ROI}$ in the left hippocampus during the prefrontal cortex stimulation ( $100 \%$ rMT minus rest over prefrontal cortex) for the placebo day and the LTG day. Compared with placebo, LTG significantly increased the number of active voxels in the left hippocampus (Wilcoxon's nonparametric test: $z=1.99, \mathrm{df}=7, p=0.04$ )

\section{Effects of LTG on rMT and BOLD Response During TMS Over the Motor Cortex}

Several prior TMS studies have shown that LTG increases the threshold of MEPs elicited by TMS (Manganotti et al, 1999; Villetti et al, 2001; Ziemann et al, 1996). In the present study, we confirmed the inhibitory effect of LTG on MEPs. LTG caused a $14.9 \%$ increase in rMT in healthy young adults, which agrees with previous TMS studies with the compound (Manganotti et al, 1999; Ziemann et al, 1998a, 1996). The effects of LTG on rMT have been suggested to be due to the inhibition of voltage-gated $\mathrm{Na}^{+}$and $\mathrm{Ca}^{2+}$ ion channels (Boroojerdi et al, 2001). However, the location of these effects cannot be conclusively determined using the technique used in that study. For example, an effect of LTG at the spinal level, rather than within the motor cortex, cannot be entirely excluded as an explanation for the drugs' observed influence on rMT (Boroojerdi et al, 2001).

Compared to a purely EMG-based study, the current approach is in a better position to detect central effects of LTG, since a direct measure of CNS activation was carried out. Indeed, the interleaved TMS/fMRI data showed that LTG reduced activation in the motor cortex, directly under the coil, and in other diffuse areas of the cortex. However, it is possible that the observed central BOLD signal could arise due to activation following sensory feedback to the motor cortex from activation of the thumb, rather than as a direct result of central activation by the applied TMS. Thus, the decrease in BOLD response in the presence of LTG may be due to an effect on the MEP, rather than a local effect in the CNS. The correlation between the increase in MEP threshold and the decrease in BOLD-fMRI measure in the presence of LTG is equally consistent with this explanation.

\section{BOLD Response to TMS Over the Prefrontal Cortex}

In addition to TMS over the motor cortex, we applied the interleaved TMS/fMRI technique with TMS over the prefrontal cortex, using a probabilistic positioning method for the TMS coil. In this case, we were limited to examining the fMRI measurements alone, since there is no overt behavioral response, such as an MEP, to assess the effect of prefrontal cortical stimulation. We have shown previously that unilateral TMS applied over the prefrontal cortex (left) has bilateral effects and that higher intensity stimulation produces greater ipsi- and contralateral activation (Nahas et al, 2001). In addition, other PET and SPECT studies have shown that increases and decreases in blood flow or metabolism occur during and shortly after repetitive TMS (rTMS) applied over the prefrontal cortex (Kimbrell et al, 

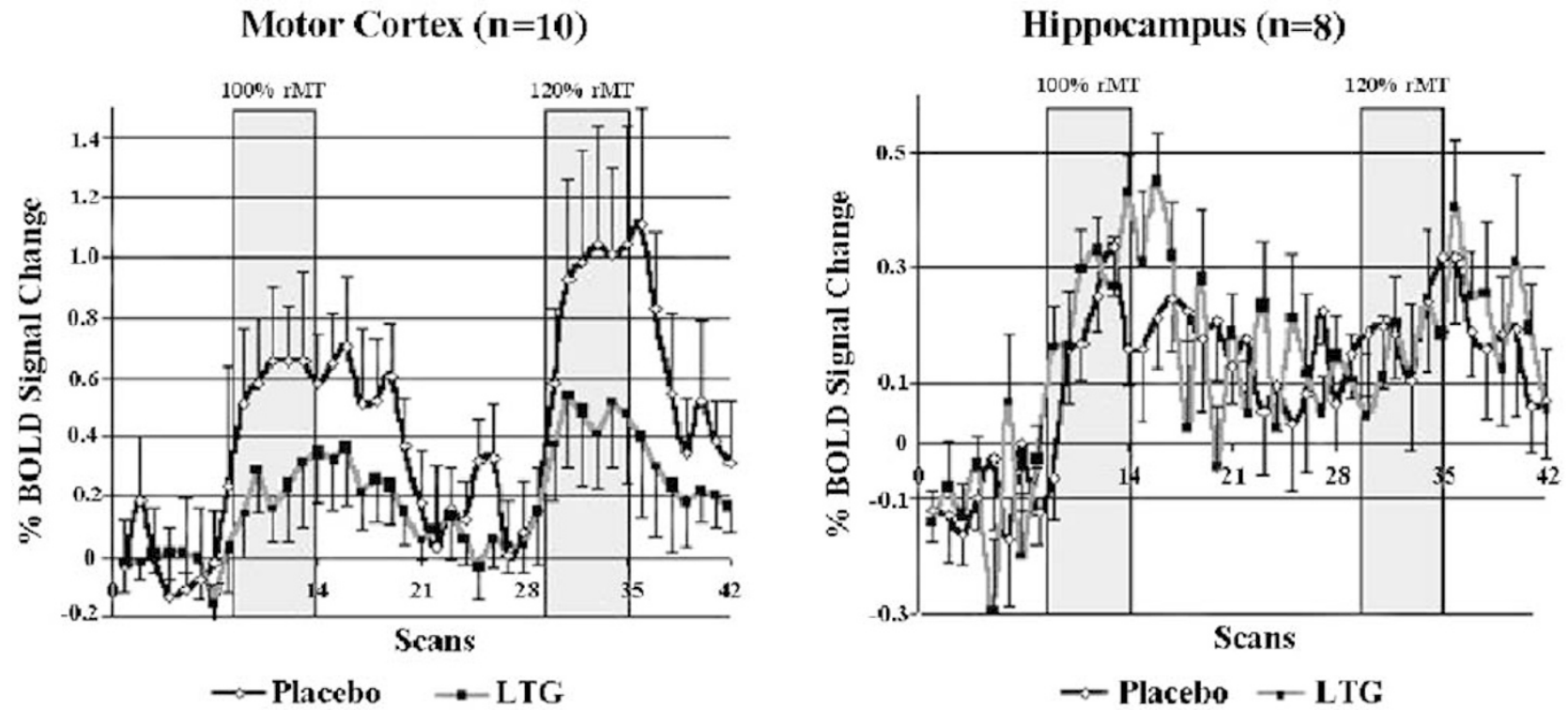

Figure 4 Average time series for TMS activation on LTG and placebo. On the left are cycle-averaged percent change in BOLD signal from baseline over time curves averaged over all 10 subjects from a voxel cluster in the left primary motor cortex directly beneath the TMS coil, during the motor cortex stimulation run. LTG diffusely inhibits the motor cortex TMS-induced activation percent change in BOLD (two-way ANOVA results showed that \% BOLD signal change of LTG significantly decreased activity compared with placebo $\left(F_{1,20}=11.89, p=0.007\right)$ and \% BOLD signal change of I20\% rMT significantly increased compared with $100 \% \mathrm{rMT}\left(\mathrm{F}_{1,6}=6.27, p=0.034\right)$. On the right are similar time series from the prefrontal interleaved TMS/fMRI run, except these are averaged over eight subjects from a voxel cluster in the left hippocampus. LTG increased the TMS-induced percent change in BOLD in this hippocampal region. Although the whole-brain $\mathrm{AMRI}$ results found this region significantly increased on LTG compared with placebo, this time series failed to reach statistic significance, considering the active time series (two-way ANOVA results failed to show significant \% BOLD signal change between either LTG and placebo $\left(F_{1,20}=1.12 p=0.326\right)$ or $100 \%$ rMT stimulation and $120 \%$ rMT stimulation $\left.\left(F_{1,6}=0.32, p=0.59 \mid\right)\right)$.

1999; Speer et al, 2000; Strafella et al, 2001). The present study confirmed the bilateral cortical activation following TMS over the prefrontal cortex. However, we found no significant difference between the activation at $100 \%$ rMT and activation at $120 \%$ rMT (in the presence of placebo). Interestingly, in contrast to motor cortex TMS, as well as our previous findings with prefrontal TMS (Nahas et al, 2001), we also failed to observe activation of the cortex directly underneath the coil. A possible explanation for this could relate to the absence of sensory feedback in the case of prefrontal stimulation.

\section{Effects of LTG on the BOLD Response During TMS Over the Prefrontal Cortex}

In the presence of LTG, we observed significant bilateral activation of the limbic system, which was not observed after placebo. This result, where LTG is not inhibiting but rather increasing (limbic) activation, subtly suggests that LTG may have unique effects on the limbic system, which differ from its effects on the motor circuits. This may be due to differential regional effects of LTG or due to some interaction of cortical-limbic loops and relative governance. However, although these are intriguing results, they are highly speculative given the nonhypothesized nature of the findings and the small sample size. An additional study attempting replication is needed.

\section{Mechanism of Action of LTG}

A key finding of the present study was that BOLD responses induced by TMS in the motor cortex could be inhibited by
LTG (a BOLD signal decreased of $50 \%$ relative to baseline) and that this decrease correlated significantly with the increased $\mathrm{rMT}$ in the presence of the drug. In addition, the effect of LTG was stronger on TMS applied at $120 \%$ rMT than at $100 \% \mathrm{rMT}$, and the time-series analysis (Figure 4 ) suggests a greater effect of LTG towards the end of the series of stimulations. Similar effects of LTG on BOLD fMRI activation have been reported previously (Kida et al, 2001) during a study of the activation of somatosensory cortex following foot-pinch in anesthetized rats. LTG is a usedependent inhibitor of brain sodium channels (Catterall 1999; Xie et al, 1995) and it has also been suggested to reduce glutamate release and increase GABA release under some circumstances (Cunningham and Jones, 2000; Ketter et al, 2003; Waldmeier et al, 1996). These actions are consistent with the observations in the present study, although further studies using other approaches would be required to determine whether one or other mechanism is more important.

\section{What Might This Study Tell us About LTG's Mechanism of Action in Bipolar Disorder?}

Double-blind, placebo-controlled trials have demonstrated the acute and prophylactic antidepressant activity of LTG in bipolar disorder (Calabrese, 1999; Calabrese et al, 1999; Ketter and Calabrese, 2002). Various hypotheses have been proposed with regard to its mechanism of action on mood. One may speculate that the efficacy of LTG in bipolar disorder is related to its anticonvulsant efficacy and so also to its anticonvulsant mechanisms of action. However, the clinical profile of LTG in bipolar disorder is different from 
that of another anticonvulsant mood stabilizer, carbamazepine. In fact, LTG's spectrum of anticonvulsant efficacy is also somewhat different, notably with respect to its unique efficacy $v s$ absence of seizures (Muzina et al, 2002).

The present study in healthy volunteers may not be relevant to drug effects in patients with bipolar disorder, but the surprising observation of limbic activation in the presence of LTG when TMS was applied to the prefrontal cortex is worth considering with the clinical situation in mind. Studies of the neuropathology in familial Major Depressive Disorder have reported changes in morphology and metabolism in selected areas of the limbic system, such as the hippocampus (Auer et al, 2000; Rusch et al, 2001; Thibault et al, 2001), the orbital frontal lobe (Drevets 2000; MacFall et al, 2001), and amygdala (Sheline et al, 1998). Frodl et al (2002) reported smaller hippocampal gray matter volumes in patients with a first episode of major depression compared with healthy subjects. Furthermore, recent data suggest (Heckers et al, 2002) that bipolar disorder is associated with a significant decrease of glutamic acid decarboxylase (GAD) mRNA-positive neurons and of $\mathrm{GAD}_{65}$ mRNA expression in the hippocampus. Pharmacotherapeutic studies have also reported increased regional activation of the left prefrontal cortex, thalamus, and medial frontal gyrus post-treatment in depressed patients (Baxter et al, 1989; Bench et al, 1995; Buchsbaum et al, 1997; Mayberg et al, 1999; Saxena et al, 2002). Taken together, these findings indicate that there are abnormalities of the limbic system in depressed patients. The present study showed that LTG could facilitate increased activation of the hippocampus after TMS over the prefrontal cortex in normal subjects.

Several previous studies have shown that TMS over the prefrontal cortex can cause changes in activity in the hippocampus of humans (Kimbrell et al, 2002; Speer et al, 2000) and animals (Levkovitz et al, 2001; Levkovitz and Segal, 2001). Functional interaction between the two areas is well documented. For example, Alexander et al (1986) originally described five parallel circuits that link the cortex with the basal ganglia. This includes a connection from the prefrontal cortex to the hippocampus. Furthermore, prefrontal stimulation has been shown to produce an increase in serotonin (5-HT) in the hippocampus (Groenewegen et al, 1997; Juckel et al, 1999). Interestingly, LTG has been shown to inhibit the uptake of serotonin, norepinephrine, and dopamine by rat cortical synaptosomes in vitro (Southam et al, 1998), although changes in monoamine levels could not be observed in vivo (Xie and Hagan, 1998). Further study will be required to confirm and elucidate the changes in limbic activation seen in the present study.

\section{Limitations of the Present Study}

This initial proof-of-concept study suffers from limitations that bear on the interpretation of the results. There is active debate about whether subthreshold TMS at $1 \mathrm{~Hz}$ over the motor cortex produces activation or inhibition. Embedded in this discussion is concern about whether and how much of the blood flow changes seen might be due, not to direct activation produced by the TMS, but by indirect sensory feedback produced by the muscle movement that TMS causes (Baudewig et al, 2001). One interpretation of the current results is therefore, that LTG causes an increase in rMT leading to less activation of the target muscle for a given stimulus, and so less sensory feedback to motor cortex resulting in less activation observed in the BOLD signal. Further work is needed to settle this important and complex issue. One possible approach might be to apply a local anesthetic to the target muscle to help to distinguish direct from indirect effects of the TMS on the CNS.

A second issue that follows partly from the above was the decision to use the same stimulation amplitude (with the Dantec TMS) during the fMRI acquisition on the second visit as that during the first visit, even though clearly the rMT would be different due to the presence or absence of LTG. The alternative would have been to redetermine the rMT using the Dantec TMS just prior to fMRI acquisition on the second visit, which would likely mean that a higher TMS intensity would be used when subjects received LTG (assuming that LTG raises rMT). We felt that it was better to deliver the same stimulus on each visit so that any change in BOLD signal would be due only to the presence or absence of drug, thus simplifying interpretation of the data. As a result, those receiving placebo on the first visit would be relatively understimulated on the second visit when LTG was given, and vice versa. Since the study was conducted in a double-blind, crossover design, those subjects who were 'understimulated' on the second visit would be balanced by those who were 'overstimulated'. Importantly, a comparison of test order showed that whether LTG was administrated on the first visit or the second visit, it produced a similar effect on rMT.

The prefrontal cortex data are potentially easier to interpret than the motor cortex data, since the issue of whether the BOLD signal might be due to sensory feedback rather than direct activation is presumably absent. However, while the pattern of activation following TMS over the motor cortex replicated our previous work, the prefrontal TMS data failed to replicate our earlier finding of activation of the cortex directly underneath the coil (Nahas et al, 2001). In addition, although the present data from the motor cortex TMS showed that higher-intensity stimulation produced greater activation in the cortex under the stimulation site, we failed to find an intensity-dependent brain activation when stimulation was applied over the prefrontal cortex (Figure 4). The nature of the interaction of the TMS signal with nervous tissue directly under the stimulation site remains largely unknown; the present results serve only to encourage further research into this critical issue.

Finally, our subjects were healthy adults, and the findings cannot necessarily be generalized to patients with mood disorders. This was also a single dose challenge study and different effects may occur with chronic dosing. Once the present study has been replicated in healthy volunteers, longitudinal studies in patients undergoing drug therapy would be warranted.

\section{CONCLUSIONS}

In conclusion, this current study suggests that the interleaved TMS/fMRI technique has potential utility in understanding the regional brain effects of LTG and other 
CNS-active compounds. Using the technique, we found, as hypothesized, that LTG has an inhibitory effect on motor circuit excitability measured both by rMT and interleaved TMS/fMRI. The study also found that LTG has a complex effect on response to TMS applied over the prefrontal cortex, causing cortical inhibition and limbic facilitation. It is unclear if these effects, which did not correlate with rMT, may be relevant to the efficacy of LTG in mood disorders. Further studies are warranted with this promising but complex new technique.

\section{ACKNOWLEDGEMENTS}

This study was funded primarily by an unrestricted research grant from GSK to Dr George as well as from Center for Advanced Imaging Research and Brain Stimulation Laboratory infrastructure and resources. We would like to acknowledge the help of Poya Moghadam (MUSC summer student) as well as helpful past discussions with Barbara Grassi of GSK. The authors and the Brain Stimulation Laboratory are supported in part by research grants from the National Alliance for Research on Schizophrenia and Depression (NARSAD), the Stanley Foundation, NINDS Grant RO1-AG40956, and the Defense Advanced Research Projects Agency (DARPA). The Laboratory has also received grant support from Cyberonics (VNS), Neotonus, and Neuronetics (TMS) for clinical trials. None of the authors have equity or financial conflicts. Drs George, Nahas, and Bohning have filed an invention disclosure for interleaving TMS with fMRI as a tool for examining the brain effects of CNS compounds. This work was presented in abstract form at the Annual Meeting of American College of Neuropsychopharmacology, Hawaii, December 14, 2001 and the Annual Meeting of American Psychiatric Association, Philadelphia, PA, May 21, 2002.

\section{REFERENCES}

Alexander GE, DeLong MR, Strick PL (1986). Parallel organization of functionally segregated circuits linking basal ganglia and cortex. Annu Rev Neurosci 9: 357-381.

Annett M (1970). A classification of hand preference by association analysis. Br J Psychol 61: 303-321.

Auer DP, Putz B, Kraft E, Lipinski B, Schill J, Holsboer F (2000). Reduced glutamate in the anterior cingulate cortex in depression: an in vivo proton magnetic resonance spectroscopy study. Biol Psychiatry 47: 305-313.

Baudewig J, Siebner HR, Bestmann S, Tergau F, Tings T, Paulus W et al (2001). Functional MRI of cortical activations induced by transcranial magnetic stimulation (TMS). Neuroreport 12: 3543-3548.

Baxter Jr LR, Schwartz JM, Phelps ME, Mazziotta JC, Guze BH, Selin CE et al (1989). Reduction of prefrontal cortex glucose metabolism common to three types of depression. Arch Gen Psychiatry 46: 243-250.

Bench CJ, Frackowiak RS, Dolan RJ (1995). Changes in regional cerebral blood flow on recovery from depression. Psychol Med 25: 247-261.

Bestmann S, Baudewig J, Frahm J (2003). On the synchronization of transcranial magnetic stimulation and functional echo-planar imaging. J Magn Reson Imaging 17: 309-316.

Bohning DE, Denslow S, Bohning PA, Walker JA, George MS (2003). A TMS coil positioning/holding system for MR image- guided TMS interleaved with fMRI. Clin Neurophysiol 114: 2210-2219.

Bohning DE, He L, George MS, Epstein CM (2001). Deconvolution of transcranial magnetic stimulation (TMS) maps. J Neural Transm 108: 35-52.

Bohning DE, Shastri A, McConnell KA, Nahas Z, Lorberbaum JP, Roberts DR et al (1999). A combined TMS/fMRI study of intensity-dependent TMS over motor cortex. Biol Psychiatry 45: 385-394.

Bohning DE, Shastri A, McGavin L, McConnell KA, Nahas Z, Lorberbaum JP et al (2000). Motor cortex brain activity induced by $1-\mathrm{Hz}$ transcranial magnetic stimulation is similar in location and level to that for volitional movement. Invest Radiol 35: 676-683.

Boroojerdi B, Battaglia F, Muellbacher W, Cohen LG (2001). Mechanisms influencing stimulus-response properties of the human corticospinal system. Clin Neurophysiol 112: 931-937.

Boroojerdi B, Foltys H, Krings T, Spetzger U, Thron A, Topper R (1999). Localization of the motor hand area using transcranial magnetic stimulation and functional magnetic resonance imaging. Clin Neurophysiol 110: 699-704.

Buchsbaum MS, Wu J, Siegel BV, Hackett E, Trenary M, Abel L et al (1997). Effect of sertraline on regional metabolic rate in patients with affective disorder. Biol Psychiatry 41: 15-22.

Calabrese JR (1999). Lamotrigine and the treatment of bipolar disorder. Introduction. Eur Neuropsychopharmacol 9(Suppl 4 AD): S107-S108.

Calabrese JR, Bowden CL, Sachs GS, Ascher JA, Monaghan E, Rudd GD (1999). A double-blind placebo-controlled study of lamotrigine monotherapy in outpatients with bipolar I depression. Lamictal 602 Study Group. J Clin Psychiatry 60: 79-88.

Calabrese JR, Gajwani P (2000). Lamotrigi ne and clozapine for bipolar disorder. Am J Psychiatry 157: 1523.

Catterall WA (1999). Molecular properties of brain sodium channels: an important target for anticonvulsant drugs. $A d v$ Neurol 79: 441-456.

Cunningham MO, Jones RS (2000). The anticonvulsant, lamotrigine decreases spontaneous glutamate release but increases spontaneous GABA release in the rat entorhinal cortex in vitro. Neuropharmacology 39: 2139-2146.

Dassonville P, Lewis SM, Zhu XH, Ugurbil K, Kim SG, Ashe J (1998). Effects of movement predictability on cortical motor activation. Neurosci Res 32: 65-74.

Drevets WC (2000). Functional anatomical abnormalities in limbic and prefrontal cortical structures in major depression. Prog Brain Res 126: 413-431.

First SR MB, Gibbon M, Williams JBW (1995). Structured Clinical Interview for DSM-IV I Disorder. American Psychiatric Association: Washington, DC.

Frodl T, Meisenzahl EM, Zetzsche T, Born C, Groll C, Jager M et al (2002). Hippocampal changes in patients with a first episode of major depression. Am J Psychiatry 159: 1112-1118.

Frye MA, Ketter TA, Kimbrell TA, Dunn RT, Speer AM, Osuch EA et al (2000). A placebo-controlled study of lamotrigine and gabapentin monotherapy in refractory mood disorders. J Clin Psychopharmacol 20: 607-614.

George MS, Lisanby SH, Sackeim HA (1999). Transcranial magnetic stimulation: applications in neuropsychiatry. Arch Gen Psychiatry 56: 300-311.

George MS, Wassermann EM, Williams WA, Callahan A, Ketter TA, Basser P et al (1995). Daily repetitive transcranial magnetic stimulation (rTMS) improves mood in depression. Neuroreport 6: $1853-1856$.

Groenewegen HJ, Wright CI, Uylings HB (1997). The anatomical relationships of the prefrontal cortex with limbic structures and the basal ganglia. J Psychopharmacol 11: 99-106.

Heckers S, Stone D, Walsh J, Shick J, Koul P, Benes FM (2002). Differential hippocampal expression of glutamic acid 
decarboxylase 65 and 67 messenger RNA in bipolar disorder and schizophrenia. Arch Gen Psychiatry 59: 521-529.

Hlustik P, Solodkin A, Gullapalli RP, Noll DC, Small SL (2002). Functional lateralization of the human premotor cortex during sequential movements. Brain Cogn 49: 54-62.

Juckel G, Mendlin A, Jacobs BL (1999). Electrical stimulation of rat medial prefrontal cortex enhances forebrain serotonin output: implications for electroconvulsive therapy and transcranial magnetic stimulation in depression. Neuropsychopharmacology 21: 391-398.

Ketter TA, Calabrese JR (2002). Stabilization of mood from below versus above baseline in bipolar disorder: a new nomenclature. J Clin Psychiatry 63: 146-151.

Ketter TA, Manji HK, Post RM (2003). Potential mechanisms of action of lamotrigine in the treatment of bipolar disorders. J Clin Psychopharmacol 23: 484-495.

Kida I, Hyder F, Behar KL (2001). Inhibition of voltage-dependent sodium channels suppresses the functional magnetic resonance imaging response to forepaw somatosensory activation in the rodent. J Cereb Blood Flow Metab 21: 585-591.

Kimbrell TA, Dunn RT, George MS, Danielson AL, Willis MW, Repella JD et al (2002). Left prefrontal-repetitive transcranial magnetic stimulation (rTMS) and regional cerebral glucose metabolism in normal volunteers. Psychiatry Res 115: 101-113.

Kimbrell TA, Little JT, Dunn RT, Frye MA, Greenberg BD, Wassermann EM et al (1999). Frequency dependence of antidepressant response to left prefrontal repetitive transcranial magnetic stimulation (rTMS) as a function of baseline cerebral glucose metabolism. Biol Psychiatry 46: 1603-1613.

Levkovitz Y, Grisaru N, Segal M (2001). Transcranial magnetic stimulation and antidepressive drugs share similar cellular effects in rat hippocampus. Neuropsychopharmacology 24: 608-616.

Levkovitz Y, Segal M (2001). Aging affects transcranial magnetic modulation of hippocampal evoked potentials. Neurobiol Aging 22: 255-263.

MacFall JR, Payne ME, Provenzale JE, Krishnan KR (2001). Medial orbital frontal lesions in late-onset depression. Biol Psychiatry 49: 803-806.

Manganotti P, Bongiovanni LG, Zanette G, Turazzini M, Fiaschi A (1999). Cortical excitability in patients after loading doses of lamotrigine: a study with magnetic brain stimulation. Epilepsia 40: 316-321.

Mayberg HS, Liotti M, Brannan SK, McGinnis S, Mahurin RK, Jerabek PA et al (1999). Reciprocal limbic-cortical function and negative mood: converging PET findings in depression and normal sadness. Am J Psychiatry 156: 675-682.

Messenheimer JA (1995). Lamotrigine. Epilepsia 36(Suppl 2): S87-S94.

Mills KR, Kimiskidis V (1996). Motor cortex excitability during ballistic forearm and finger movements. Muscle Nerve 19: 468-473.

Muzina DJ, El-Sayegh S, Calabrese JR (2002). Antiepileptic drugs in psychiatry - focus on randomized controlled trial. Epilepsy Res 50: 195-202.

Nahas Z, Lomarev M, Roberts DR, Shastri A, Lorberbaum JP, Teneback C et al (2001). Unilateral left prefrontal transcranial magnetic stimulation (TMS) produces intensity-dependent bilateral effects as measured by interleaved BOLD fMRI. Biol Psychiatry 50: 712-720.

Paus T, Jech R, Thompson CJ, Comeau R, Peters T, Evans AC (1997). Transcranial magnetic stimulation during positron emission tomography: a new method for studying connectivity of the human cerebral cortex. J Neurosci 17: 3178-3184.

Paus T, Wolforth M (1998). Transcranial magnetic stimulation during PET: reaching and verifying the target site. Hum Brain Mapp 6: 399-402.

Rusch BD, Abercrombie HC, Oakes TR, Schaefer SM, Davidson RJ (2001). Hippocampal morphometry in depressed patients and control subjects: relations to anxiety symptoms. Biol Psychiatry 50: $960-964$.

Saxena S, Brody AL, Ho ML, Alborzian S, Maidment KM, Zohrabi $\mathrm{N}$ et al (2002). Differential cerebral metabolic changes with paroxetine treatment of obsessive-compulsive disorder $v s$ major depression. Arch Gen Psychiatry 59: 250-261.

Shastri A, George MS, Bohning DE (1999). Performance of a system for interleaving transcranial magnetic stimulation with steady-state magnetic resonance imaging. Electroencephalogr Clin Neurophysiol Suppl 51: 55-64.

Sheline YI, Gado MH, Price JL (1998). Amygdala core nuclei volumes are decreased in recurrent major depression. Neuroreport 9: 2023-2028.

Smith MJ, Adams LF, Schmidt PJ, Rubinow DR, Wassermann EM (2002). Effects of ovarian hormones on human cortical excitability. Ann Neurol 51: 599-603.

Solodkin A, Hlustik P, Noll DC, Small SL (2001). Lateralization of motor circuits and handedness during finger movements. Eur J Neurol 8: 425-434.

Southam E, Kirkby D, Higgins GA, Hagan RM (1998). Lamotrigine inhibits monoamine uptake in vitro and modulates 5-hydroxytryptamine uptake in rats. Eur J Pharmacol 358: 19-24.

Speer AM, Kimbrell TA, Wassermann EM, Repella D, Willis MW, Herscovitch P et al (2000). Opposite effects of high and low frequency rTMS on regional brain activity in depressed patients. Biol Psychiatry 48: 1133-1141.

Strafella AP, Paus T, Barrett J, Dagher A (2001). Repetitive transcranial magnetic stimulation of the human prefrontal cortex induces dopamine release in the caudate nucleus. $J$ Neurosci 21: RC157.

Tergau F, Wischer S, Somal HS, Nitsche MA, Joe Mercer A, Paulus W et al (2003). Relationship between lamotrigine oral dose, serum level and its inhibitory effect on CNS: insights from transcranial magnetic stimulation. Epilepsy Res 56: $67-77$.

Thibault O, Hadley R, Landfield PW (2001). Elevated postsynaptic $\left[\mathrm{Ca}^{2+}\right]_{\mathrm{i}}$ and L-type calcium channel activity in aged hippocampal neurons: relationship to impaired synaptic plasticity. J Neurosci 21: 9744-9756.

Villetti G, Bregola G, Bassani F, Bergamaschi M, Rondelli I, Pietra C et al (2001). Preclinical evaluation of CHF3381 as a novel antiepileptic agent. Neuropharmacology 40: 866-878.

Waldmeier PC, Martin P, Stocklin K, Portet C, Schmutz M (1996). Effect of carbamazepine, oxcarbazepine and lamotrigine on the increase in extracellular glutamate elicited by veratridine in rat cortex and striatum. Naunyn Schmiedebergs Arch Pharmacol 354: 164-172.

Xie X, Hagan RM (1998). Cellular and molecular actions of lamotrigine: possible mechanisms of efficacy in bipolar disorder. Neuropsychobiology 38: 119-130.

Xie X, Lancaster B, Peakman T, Garthwaite J (1995). Interaction of the antiepileptic drug lamotrigine with recombinant rat brain type IIA $\mathrm{Na}^{+}$channels and with native $\mathrm{Na}^{+}$channels in rat hippocampal neurones. Pflugers Arch 430: 437-446.

Ziemann U, Hallett M, Cohen LG (1998a). Mechanisms of deafferentation-induced plasticity in human motor cortex. J Neurosci 18: 7000-7007.

Ziemann U, Lonnecker S, Steinhoff BJ, Paulus W (1996). Effects of antiepileptic drugs on motor cortex excitability in humans: a transcranial magnetic stimulation study. Ann Neurol 40: 367-378.

Ziemann U, Steinhoff BJ, Tergau F, Paulus W (1998b). Transcranial magnetic stimulation: its current role in epilepsy research. Epilepsy Res 30: 11-30.

Ziemann U, Tam A, Butefisch C, Cohen LG (2002). Dual modulating effects of amphetamine on neuronal excitability and stimulation-induced plasticity in human motor cortex. Clin Neurophysiol 113: 1308-1315. 